\title{
Correlation analysis on the expression levels of microRNA-23a and microRNA-23b and the incidence and prognosis of ovarian cancer
}

\author{
$\mathrm{LI} \mathrm{SU}^{1}$ and MINGMEI LIU ${ }^{2}$ \\ ${ }^{1}$ Department of Obstetrics and Gynecology, Longnan Hospital; ${ }^{2}$ Department of Obstetrics and Gynecology, \\ Oilfields General Hospital in Daqing, Daqing, Heilongjiang 163001, P.R. China
}

Received August 25, 2017; Accepted December 10, 2017

DOI: 10.3892/ol.2018.8669

\begin{abstract}
The present study aimed to investigate the expression levels of microRNA (miR)-23a and miR-23b in the tumor tissues of patients with ovarian cancer. The study also explored the correlation of miR-23a and miR-23b expression levels in the tumor tissues with the clinic-pathological parameters and prognosis of the patients. Specimens of frozen tumor tissues and normal tissues adjacent to the tumor were collected from 50 patients with ovarian cancer. Reverse transcription-quantitative polymerase chain reaction was adopted to detect the expression levels of miR-23a and miR-23b in tumor tissues. Furthermore, normal tissues adjacent to the tumor were utilized as the control for the experiments and Pearson method was used to analyze the correlation between miR-23a and miR-23b expression levels in tumor tissues. The correlation of miR-23a and miR-23 expression in tumor tissues and the prognosis of the patients with ovarian cancer was analyzed in combination with clinical data. The expression of miR-23a in the tissues of ovarian cancer was significantly higher in comparison with normal adjacent tissues. However, the expression of miR-23b in the tissues of ovarian cancer was significantly lower when compared with adjacent normal tissues. Notably, the expression of miR-23a was negatively correlated with that of miR-23b in tumor tissues of ovarian cancer. The high expression of miR-23a and the low expression of miR-23b in tumor tissues of the patients was correlated with the degree of tumor differentiation, metastasis of lymph nodes and clinical staging. The five-year overall survival rate of the patients was $36 \%$ (18/50). Univariate survival analysis indicated that miR-23a and $\mathrm{miR}-23 \mathrm{~b}$ were the factors influencing the overall survival rate of ovarian cancer. The present findings suggest that high expression of miR-23a and the low expression of miR-23b are closely correlated with the occurrence and development of ovarian cancer. The abnormal expression of the miR-23a and
\end{abstract}

Correspondence to: Dr Mingmei Liu, Department of Obstetrics and Gynecology, Oilfields General Hospital in Daqing, 9 Zhongkang Street, Daqing, Heilongjiang 163001, P.R. China

E-mail: shdgf147@163.com

Key words: ovarian cancer, microRNA-23a, microRNA-23b, prognosis
miR-23b could be utilized as potential prognostic molecular markers of ovarian cancer.

\section{Introduction}

Ovarian cancer is a common malignant tumor in female genital system and its incidence rate ranks second in the malignant tumors of female genital system. Moreover, its mortality has been ranked as first in female malignant tumors. Approximately $85 \%$ of the patient has been reported to be suffering from epithelial ovarian cancer (1). At present, ovarian cancer is mainly treated with comprehensive treatment methods including surgery, chemotherapy, radiotherapy, biological therapy, etc., clinically. Although the methods for diagnosis and treatment of ovarian cancer is being developed continuously, the 5-year survival rate of the patients is still just $30 \%$. Therefore, it is of great clinical significance to search molecular markers for early diagnosis of ovarian cancer and for the judgment of prognosis (2).

MicroRNA (miRNA/miR) is a small highly-conserved, single-stranded and non-coding RNA, which could be specifically associated with its target mRNA, as it affects directly the transcription or translation processes of the target genes $(3,4)$. It has been confirmed in an earlier study that the abnormal expression of miRNA in tumor cells is responsible for the development of various malignant tumors (5). miR-23 has two subtypes: miR-23a and miR-23b. They only differ by one nucleotide and are coded by different genes (6). miR-23a is located on chromosome 19 . It not only regulates the growth and differentiation of normal cells, but also controls the proliferation as well as metastasis of a variety of malignant tumor cells $(7,8)$. miR-23b is located on chromosome 9 and its abnormal expression has been noticed in prostate cancer, pancreatic cancer, liver cancer and other malignant tumors. Moreover, miR-23b has an influence on the pathology and prognosis of the patients (9).

Currently, there is neither any study on the correlation between the expressions of miR-23a and miR-23b during ovarian cancer nor the any study about their influence on the pathological parameters and prognosis of ovarian cancer. Therefore, reverse transcription-quantitative polymerase chain reaction (RT-qPCR) was adopted in this study to detect the expressions of miR-23a and miR-23b in tumor tissues of patients with ovarian cancer. The prime aim of the study was to investigate the correlation between the expressions of the 
two items. Meanwhile, the influences of miR-23a and miR-23b in tumor tissues of the patients on the pathological parameters and prognosis of patients with ovarian cancer were studied in combination with the analysis of clinical data.

\section{Materials and methods}

Study design. A total of 50 patients who were treated in surgical department of our hospital from June 2008 to December 2011 were enrolled as study subjects in this study. The study subjects aged 28-76 years old and had an average age of 54.8. Tumor tissues and normal tissues adjacent to the cancer of the patients with ovarian cancer were taken during surgery and were preserved in liquid nitrogen. Inclusion criteria for the patients: Patients who were treated for the first time without radiotherapy or chemotherapy, and patients confirmed with ovarian epithelial cancer by pathology after surgery Exclusion criteria: patients who had a history of cardiovascular diseases, neurological diseases, and diabetes, accompanied with malignant tumors in other parts during preoperative examinations. The clinical ethics committee of our hospital had approved the present study. Further, all patients approved for the study or their families had signed the informed consent.

TRIzol kits, reverse transcription kits and RT-qPCR kits (Invitrogen; Thermo Fisher Scientific, Inc., Waltham, MA, USA); primer synthesis (Takara Biotechnology Co., Ltd., Dalian, China).

Detection of the expressions of $m i R-23 a$ and $m i R-23 b$ in tumor tissues of the ovarian cancer patients with RT-qPCR. Approximately $200 \mathrm{mg}$ of samples was taken from frozen tumor tissues and normal tissues adjacent to the cancer respectively. The total RNA in the specimens of the tissues was extracted as per the method described in the package insert of TRIzol kit to detect the absorbance of RNA at 260 and $280 \mathrm{~nm}$. Reverse transcription was conducted for the samples with A260/A280 between 1.8-2.0. The operation was carried out as per the package insert of reverse transcription kit. The-obtained cDNA was used as the template and PCR operation was carried out as per the recommended method of the kit. The total volume of the PCR reaction system was $20 \mu \mathrm{l}$ and the primer sequence was indicated in Table I. Reaction conditions: $94^{\circ} \mathrm{C}$ for $4 \mathrm{~min}$ (initial denaturation); $95^{\circ} \mathrm{C}$ for $1 \mathrm{~min}$; $60^{\circ} \mathrm{C}$ for $1 \mathrm{~min}$ and $72^{\circ} \mathrm{C}$ for $1 \mathrm{~min}$; amplification for 40 cycles. $\mathrm{Ct}$ value was output from the instrument. The results were analyzed by $2^{-\Delta \Delta \mathrm{Cq}}$ method using U6 RNA as control.

Analysis of the correlation between $t \mathrm{miR}-23 \mathrm{a}$ and $\mathrm{miR}-23 \mathrm{~b}$ expressions in tumor tissues during ovarian cancer and the pathological/prognosis parameters. The patients were divided into high-expression group and low-expression group with the median of the expressions of miR-23a (5.35) and miR-23b (2.87) in tumor tissues of the patients with ovarian cancer as the benchmark. $\chi^{2}$ test was conducted to analyze the correlation between the expressions of miR-23a and miR-23b and the pathological parameters of the patients. Follow-up visit was conducted once every month from the first day after surgery for 5 years.

Statistical analysis. SPSS 17.0 software (SPSS, Inc., Chicago, IL, USA) was used for data analyses. Further, $\chi^{2}$ test was conducted for the comparison of enumeration data between groups. Enumeration data were expressed as mean \pm standard deviation and t-test was conducted for the comparison between two groups. Pearson analysis was performed to analyze correlation, Kaplan-Meier analysis was adopted for survival analysis and log-rank method was used for the comparison between groups. $\mathrm{P}<0.05$ was considered to indicate a statistically significant difference.

\section{Results}

Detection of the expressions of miR-23a and miR-23b in tumor tissues of ovary ancer patients with RT-qPCR. RT-qPCR results are indicated in Fig. 1. miR-23a showed significantly higher expression in tumor tissues $(\mathrm{P}<0.01)$ in comparison to adjacent normal tissues. On the other hand, miR-23b revealed significantly lower expression $(\mathrm{P}<0.01)$ in tumor tissues when compared with adjacent normal tissues.

Correlation analysis on the expressions of $m i R-23 a$ and miR-23b in tumor tissues of patients with ovarian cancer. Pearson correlation analysis was conducted for the expressions of miR-23a and miR-23b in tumor tissues of patients with ovarian cancer. The results are shown in Fig. 2. The expression amount of miR-23a is negatively correlated with that of miR-23b in tumor tissues of patients with ovarian cancer $(\mathrm{r}=-0.859, \mathrm{P}<0.01)$.

Relationship between expressions of miR-23a and miR-23b in ovarian tumors tissues with clinical pathology parameters. The results are shown in Table II. $\chi^{2}$ test indicated that the high expression of miR-23a and the low expression of miR-23b in tumor tissues of the patients were related with the degree of tumor differentiation, metastasis of lymph nodes and clinical staging.

Analysis of survival condition and prognosis of the patients. The results of 5-year follow-up visit showed that 18 cases survived and 32 cases died among the 50 cases. The 5-year overall survival rate was $36 \%(18 / 50)$ and the death rate was $64 \%$ (32/50). The Kaplan-Meier survival curve of patients with ovarian cancer is shown in Fig. 3A and B. The results clearly confirmed that the patients with high expression of miR-23a or low expression of miR-23b had relatively poor prognosis. Results of univariate survival analyses are shown in Table III, which showed that both miR-23a and miR-23b had impacts on the overall survival rate of patients with ovarian cancer $(\mathrm{P}<0.01)$.

\section{Discussion}

The pathogenesis of ovarian cancer is obscured. Further, it is quite difficult to ascertain and eradicate the cancer effectively by surgery at an early stage. In addition, ovarian cancer cells easily develop tolerance to radiotherapy and chemotherapy, leading to tumor recurrence and treatment failure. Therefore, discovering tumor markers of ovarian cancer is of great significance for the diagnosis, treatment and prognosis of ovarian cancer $(10,11)$.

The normal expression of miRNA could regulate proliferation and differentiation of cells, while its abnormal expression 
Table I. Primer sequence of reverse transcription-quantitative polymerase chain reaction.

\begin{tabular}{lll}
\hline Genes & \multicolumn{1}{c}{ Direction } & \multicolumn{1}{c}{ Primer sequence } \\
\hline miR-23a & Forward primer & 5'-ATCACATTGCCAGGGATTTCC-3' \\
& Reverse primer & 5'-CCAGTGCAGGGTCCGAGGT-3' \\
miR-23b & Forward primer & 5'-CGCGGCCGCTAGTATTATGTT-3' \\
& Reverse primer & 5'-CACATTTTAAAAAACATA-3' \\
U6 & Forward primer & 5'-GCTTCGGCAGCACATATACTAAAAT-3' \\
& Reverse primer & 5'-CGCTTCACGAATTTGCGTGTCAT-3' \\
\hline
\end{tabular}

miR, microRNA.
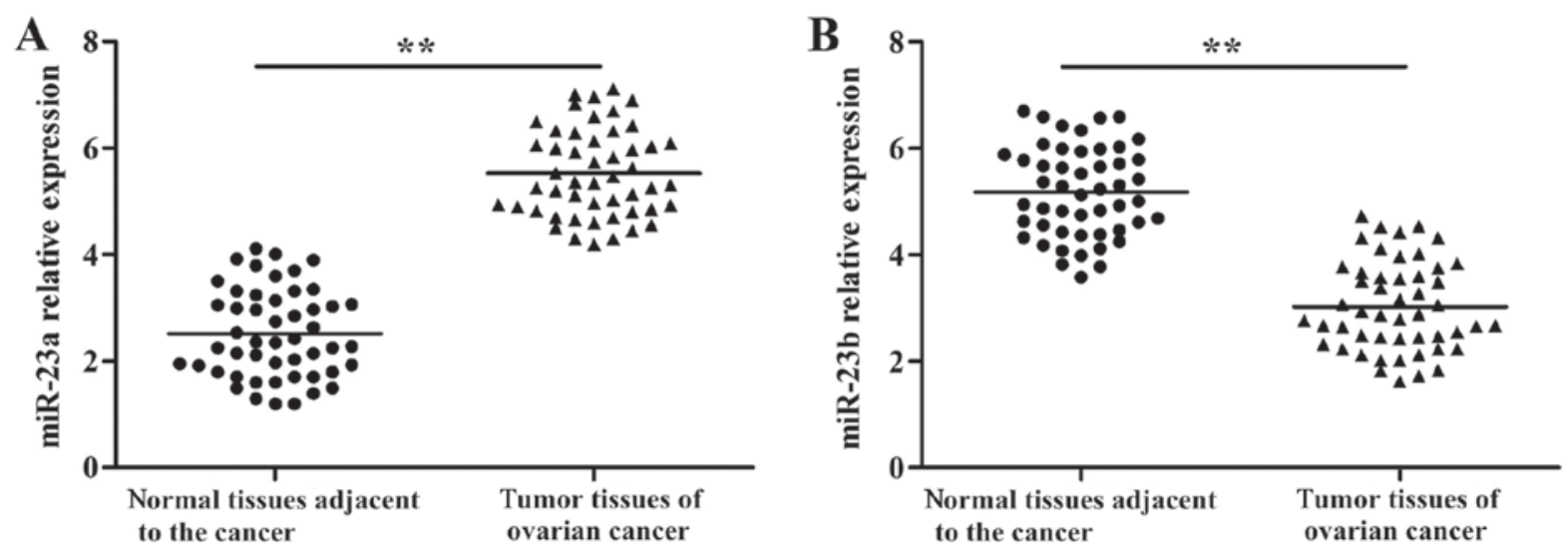

Figure 1. Detection of the expressions of miR-23a and miR-23b in tissues of the patients with RT-qPCR method. Compared with normal tissues adjacent to the cancer, miR-23a has significantly higher expression in tumor tissues of patients with ovarian cancer, while miR-23b has obviously lower expression. ${ }^{* *} \mathrm{P}<0.01$; (A) The expression of miR-23a in tissues; (B) the expression of miR-23b in tissues.

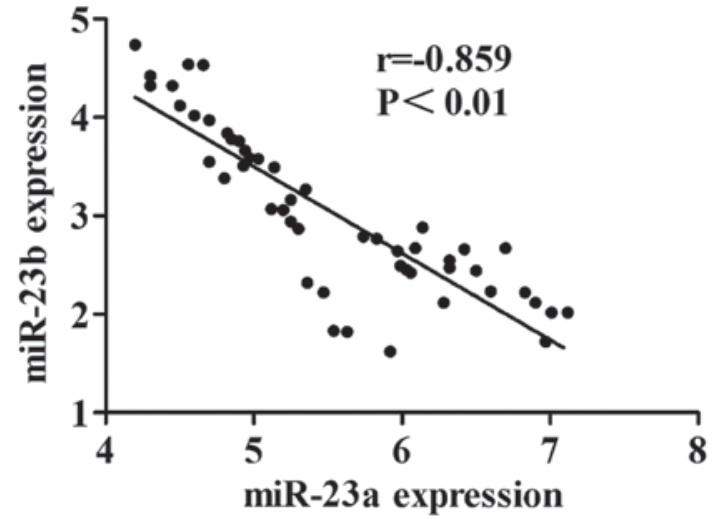

Figure 2. Correlation analysis on the expressions of miR-23a and miR-23b in tumor tissues. The expression amount of miR-23a is negatively correlated with that of miR-23b in tumor tissues of patients with ovarian cancer.

would result in development of tumors (12). It is a confirmed fact that miRNAs had an effect on the expression of target genes as they pair with the target genes, thereby, regulate various physiological processes (13). Further, it was observed in an earlier study that miRNA could regulate the expression of approximately $60 \%$ protein (14). The expression of miRNA was closely related with the growth, differentiation, multiplication, transfer and apoptosis of cells $(15,16)$.
The expressions of miR-23a and miR-23b are closely related with the proliferation, differentiation and apoptosis, etc., of the cells. Our study observed higher expression ofmir-23a in ovarian tumor tissues. miR-23a has significantly higher expression in breast cancer patients with lymph node metastasis than those without lymph node metastasis (17). MiR-23a is highly expressed in liver cancer cells, which promotes the growth and proliferation of liver cancer cells by regulating Samds signaling pathway (18). miR-23a is highly expressed in cisplatin-resistant A2780 cells of ovarian cancer. An earlier study showed that inhibition of miR-23a expression reduced the expression of P-gp protein in A2780 cells leading to elevation in sensitivity of cells to cisplatin (19). Studies have shown that miR-23b is highly expressed in the serum of patients with gastric cancer, which is negatively correlated with the prognosis of the patients (20). miR-23b showed lower expression in the tissues of ovarian cancer. When miR-23b is excessively expressed in ovarian cancer cells, the expressions of protein such as CCNG1, Survivin, Bcl-xL, P70S6K and MMP9 are decreased, thus the proliferation and migration of tumor cells are reduced (21).

In order to investigate the expressions of miR-23a and miR-23b in tumor tissues of patients with ovarian cancer and its influence on the pathological parameters and prognosis of patients with ovarian cancer, RT-qPCR was firstly adopted in this study to detect the expressions of miR-23a and miR-23b in tumor tissues of patients with ovarian cancer. The results 
Table II. Association between abnormal expression levels of miR-23a and miR-23b and the clinicopathological parameters of ovarian cancer.

Clinicopathological

parameters

Age

$\geq 50$ years old

$<50$ years old

Differentiation degree

Low differentiation

Medium/high differentiation

Metastasis of lymph nodes

Yes

No

Clinical staging

I-II

III-IV
miR-23a

miR-23a

High expression

$\mathrm{n} \quad(\mathrm{n}, \%)$

$\begin{array}{cc}\chi^{2} & \text { P-value } \\ 0.25 & >0.05\end{array}$

$\begin{array}{rr}33 & 18(54.55) \\ 17 & 8(47.06)\end{array}$

$16(48.48)$
$9(52.94)$

miR-23b

Low expression
13.88

$<0.01$

4 (19.05)

$21(72.41)$

$20(68.97)$

$11.76<0.01$

$22(70.97)$

4 (21.05)

19

5 (27.78)

$6.61<0.05$

$21(65.63)$

$\begin{array}{rcr}(\mathrm{n}, \%) & \chi^{2} & \text { P-value } \\ 16(48.48) & 0.09 & >0.05 \\ 9(52.94) & & \\ & & \\ 4(19.05) & & \\ 21(72.41) & & <0.01 \\ 23(74.19) & 19.10 & \\ 2(10.53) & & <0.01 \\ 5(27.78) & 5.56 & \\ 20(62.50) & & \end{array}$

miR, microRNA.

Table III. Univariate analysis on the correlation of the expression levels of miR-23a and miR-23b with the overall survival rate of patients with ovarian cancer.

\begin{tabular}{|c|c|c|c|c|c|}
\hline Groups & $\mathrm{n}$ & Number of survived case in 5 years & 5-year survival rate $(\%)$ & Wald (log-rank) & P-value \\
\hline \multicolumn{6}{|l|}{$\operatorname{miR}-23 a$} \\
\hline High expression & 26 & 4 & 15.38 & 16.11 & $<0.01$ \\
\hline Low expression & 24 & 14 & 58.33 & & \\
\hline \multicolumn{6}{|l|}{$\operatorname{miR}-23 b$} \\
\hline High expression & 25 & 13 & 52.00 & 12.86 & $<0.01$ \\
\hline Low expression & 25 & 5 & 20.00 & & \\
\hline
\end{tabular}

miR, microRNA.
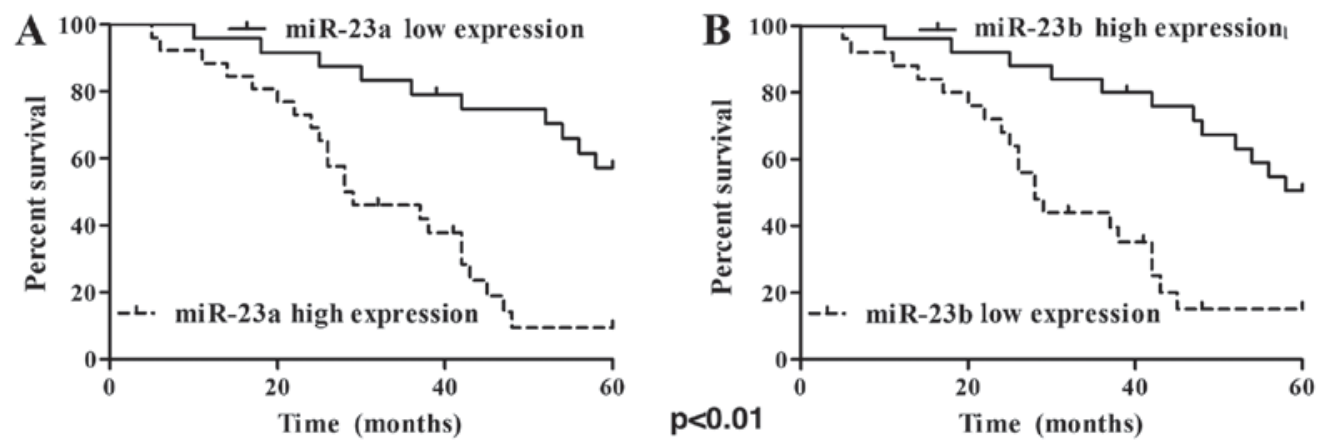

Figure 3. Kaplan-Meier survival curves of the expressions of miR-23a and miR-23b in patients with ovarian cancer. (A) Kaplan-Meier survival curves of the high and low expression of miR-23a in patients with ovarian cancer; (B) Kaplan-Meier survival curves of the high and low expression of miR-23b in patients with ovarian cancer.

showed that compared with normal tissues adjacent to the cancer, miR-23a had significantly higher expression in tissues of ovarian cancer, while the expression of miR-23b was reduced significantly. Meanwhile, Pearson correlation analysis indicated that the expression of miR-23a was negatively correlated with that of miR-23b in tumor tissues 
of patients with ovarian cancer. In order to further confirm the relationship between expressions of miR-23a and miR-23b and ovarian cancer, the correlation between the expressions of miR-23a and miR-23b and the clinical pathology/prognosis parameters was performed. The results showed that the high expression of miR-23a and the low expression of miR-23b were correlated with the degree of tumor differentiation, metastasis of lymph nodes and clinical staging. Further, survival analysis indicated that patients with high expression of miR-23a and the low expression of miR-23b had relatively poor prognosis. The univariate survival analysis showed that both miR-23a and miR-23b had a significant influence on the overall survival rate of patients with ovarian cancer. As, both miR-23a and miR-23b were negatively correlated with each other. So, there is no need of observing individual parameter with survival rate. Moreover, if in an established tumor, mir-23a was observed to be higher, and then its negative counterpart mi23b would be automatically down as a result of negative correlation. Therefore, we have studied overall survival correlation with thee two parameters collectively. Further, increase in mi23a could be called as independent prognostic marker of ovarian cancer. The above study results showed that miR-23a and miR-23b could be used as the important indicators for predicting the prognosis of patients with ovarian cancer.

In conclusion, the high expression of miR-23a and the low expression of miR-23b confirmed presence of ovarian cancer in patients. So, miR-23a and miR-23b might be utilized as important reference indicators for timely diagnosis of ovarian cancer. However, similar studies with large sample size are essential for concrete conclusion.

\section{Acknowledgements}

Not applicable.

\section{Funding}

No funding was received.

\section{Availability of data and material}

The datasets used and/or analyzed during the current study are available from the corresponding author on reasonable request.

\section{Authors' contributions}

LS wrote the manuscript and assisted with RT-qPCR. ML analyzed the survival condition and prognosis of the patients. All authors read and approved the final manuscript.

\section{Ethics approval and consent to participate}

The study was approved by the ethics committee of Longnan Hospital and informed consent was signed by the patients and/or guardians.

\section{Consent for publication}

Informed consents were signed by the patients and/or guardians.

\section{Competing interests}

The authors declare that they have no competing interests.

\section{References}

1. Siegel RL, Miller KD and Jemal A: Cancer statistics, 2015. CA Cancer J Clin 65: 5-29, 2015.

2. Siegel R, Ma J, Zou Z and Jemal A: Cancer statistics, 2014. CA Cancer J Clin 64: 9-29, 2014.

3. Panera N, Gnani D, Crudele A, Ceccarelli S, Nobili V and Alisi A: MicroRNAs as controlled systems and controllers in non-alcoholic fatty liver disease. World J Gastroenterol 20: 15079-15086, 2014.

4. Bartel DP: microRNAs: Target recognition and regulatory functions. Cell 136: 215-233, 2009.

5. Siomi $\mathrm{H}$ and Siomi MC: On the road to reading the RNA-interference code. Nature 457: 396-404, 2009.

6. Yang Z, Wang XL, Bai R, Liu WY, Li X, Liu M and Tang H: miR-23a promotes IKKa expression but suppresses ST7L expression to contribute to the malignancy of epithelial ovarian cancer cells. Br J Cancer 115: 731-740, 2016.

7. Aghaee-Bakhtiari SH, Arefian E, Naderi M, Noorbakhsh F, Nodouzi V, Asgari M, Fard-Esfahani P, Mahdian R and Soleimani M: MAPK and JAK/STAT pathways targeted by miR-23a and miR-23b in prostate cancer: Computational and in vitro approaches. Tumour Biol 36: 4203-4212, 2015.

8. Wen YC, Lee WJ, Tan P, Yang SF, Hsiao M, Lee LM and Chien MH: By inhibiting snail signaling and miR-23a-3p, osthole suppresses the EMT-mediated metastatic ability in prostate cancer. Oncotarget 6: 21120-21136, 2015.

9. Donadelli M, Dando I, Fiorini C and Palmieri M: Regulation of miR-23b expression and its dual role on ROS production and tumour development. Cancer Lett 349: 107-113, 2014.

10. Bookman MA: Optimal primary therapy of ovarian cancer. Ann Oncol 27 (Suppl 1): i58-i62, 2016.

11. Suh DH, Kim HS, Chang SJ and Bristow RE: Surgical management of recurrent ovarian cancer. Gynecol Oncol 142: 357-367, 2016.

12. Blower PE, Chung JH, Verducci JS, Lin S, Park JK, Dai Z, Liu CG, Schmittgen TD, Reinhold WC, Croce CM, et al: MicroRNAs modulate the chemosensitivity of tumor cells. Mol Cancer Ther 7: 1-9, 2008.

13. Du T and Zamore PD: microPrimer: The biogenesis and function of microRNA. Development 132: 4645-4652, 2005.

14. Lewis BP, Burge CB and Bartel DP: Conserved seed pairing, often flanked by adenosines, indicates that thousands of human genes are microRNA targets. Cell 120: 15-20, 2005.

15. Wang S and Olson EN: AngiomiRs-key regulators of angiogenesis. Curr Opin Genet Dev 19: 205-211, 2009.

16. Gounaris-Shannon S and Chevassut T: The role of miRNA in haematological malignancy. Bone Marrow Res 2013: 269107, 2013.

17. Li X, Liu X, Xu W, Zhou P, Gao P, Jiang S, Lobie PE and Zhu T: c-MYC-regulated miR-23a/24-2/27a cluster promotes mammary carcinoma cell invasion and hepatic metastasis by targeting sprouty2. J Biol Chem 288: 18121-18133, 2013.

18. Huang S, He X, Ding J, Liang L, Zhao Y, Zhang Z, Yao X, Pan Z, Zhang P, Li J, et al: Upregulation of miR-23a approximately 27 a approximately 24 decreases transforming growth factor-beta-induced tumor-suppressive activities in human hepatocellular carcinoma cells. Int J Cancer 123: 972-978, 2008.

19. Jin AH, Zhou XP and Zhou FZ: Inhibition of microRNA-23a increases cisplatin sensitivity of ovarian cancer cells: The possible molecular mechanisms. Nan Fang Yi Ke Da Xue Xue Bao 35: 125-128, 2015 (In Chinese).

20. Zhuang K, Han K, Tang H, Yin X, Zhang J, Zhang X and Zhang L: Up-regulation of plasma miR-23b is associated with poor prognosis of gastric cancer. Med Sci Monit 22: 356-361, 2016.

21. Yan J, Jiang JY, Meng XN, Xiu YL and Zong ZH: MiR-23b targets cyclin G1 and suppresses ovarian cancer tumorigenesis and progression. J Exp Clin Cancer Res 35: 31, 2016.

This work is licensed under a Creative Commons Attribution-NonCommercial-NoDerivatives 4.0 International (CC BY-NC-ND 4.0) License. 